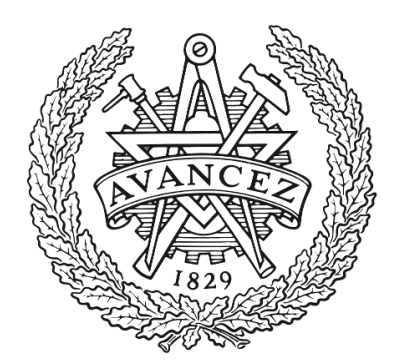

\title{
CHALMERS
}

UNIVERSITY OF TECHNOLOGY

\section{Zero-Gap Waveguide: A Parallel Plate Waveguide With Flexible Mechanical Assembly for mm-Wave Antenna Applications}

Downloaded from: https://research.chalmers.se, 2023-04-26 13:48 UTC

Citation for the original published paper (version of record):

Vosoogh, A., Uz Zaman, A., Vassilev, V. et al (2018). Zero-Gap Waveguide: A Parallel Plate Waveguide With Flexible Mechanical Assembly for mm-Wave Antenna Applications. IEEE Transactions on Components, Packaging and Manufacturing Technology, 8(12): 2052-2059. http://dx.doi.org/10.1109/TCPMT.2018.2878643

N.B. When citing this work, cite the original published paper.

C2018 IEEE. Personal use of this material is permitted.

However, permission to reprint/republish this material for advertising or promotional purposes 


\title{
Zero-gap Waveguide: A Parallel Plate Waveguide with Flexible Mechanical Assembly for mm-Wave Antenna Applications
}

\author{
Abbas Vosoogh, Ashraf Uz Zaman, Vessen Vassilev, and Jian Yang
}

\begin{abstract}
A new gap waveguide concept is presented where the air gap between the Perfect Electric Conductor (PEC) and Artificial Magnetic Conductor (AMC) parallel plates of a conventional gap waveguide structure has been reduced to almost zero. When the air gap is reduced, the periodic metal pin layer (which emulates the AMC characteristics) may come in contact with the top metal layer. A statistical analysis of a random contact of the pin texture and the top metal plate in this new type of gap technology guiding structure, so-called zero-gap, is elaborated in this work. The periodic pin texture creates a stopband for parallel plate modes, and it is also used as a mechanical support while assembling the waveguide structure. This presents a significant manufacturing advantage especially for corporate feed-network of array antennas at mm-wave frequencies. A Vband $8 \times 8$ slot array fed with this new waveguide is also designed and manufactured to show the potential of this newly proposed zero-gap waveguide structure. The measured results shows $20 \%$ of relative impedance bandwidth $\left(\left|S_{11}\right|<\mathbf{- 1 0} \mathbf{~ d B}\right)$, and an overall antenna efficiency larger than $60 \%$ over the 56.5 $69 \mathrm{GHz}$ frequency band.
\end{abstract}

Index Terms-Artificial Magnetic Conductor (AMC), Electric Discharge Machining (EDM), millimeter wave, stop-band, zerogap waveguide.

\section{INTRODUCTION}

$\mathbf{O}$ VER the past few years, the millimeter wave (mmwave) frequency band has got a lot of attention in the evolution of wireless communication systems towards $5 \mathrm{G}$ to achieve higher data rates and lower latency. Further research needs to be carried out to solve existing hardware challenges such as active and passive components integration, packaging problem and cost-effective manufacturing techniques in the development of mm-wave applications.

Planar technologies such as microstrip, coplanar waveguide (CPW), and substrate integrated waveguide (SIW) are widely used due to the interesting characteristics of low-profile, lowcost, ease of fabrication, and the best existing solutions for active components integration. However, these transmission lines have the drawbacks of high dielectric loss and field leakage, especially for increasing operating frequency. One solution to reduce the dielectric loss is to use thin-film substrate [1]. But, a narrower strip must be used in order to have a $50 \mathrm{ohm}$ line impedance, which increases the conductive loss.

This work is financially supported by the European Research Council (ERC) under the 7th Framework Program ERC grant number 321125.

A. Vosoogh, A. Uz Zaman, and J. Yang are with the Department of Electrical Engineering at Chalmers University of Technology, 41296 Gothenburg, Sweden (e-mails: \{abbas.vosoogh; zaman; jian.yang\}@ chalmers.se).

V. Vassilev is with the Microtechnology and Nanoscience Department at Chalmers University of Technology (e-mail: vessen.vassilev@chalmers.se).
Metallic hollow waveguides have benefits of low losses and high power handling capability compared to planar technologies. Fabrication and assembly issues of complex waveguide structures present a challenging task, in particular at $\mathrm{mm}$ wave frequencies. The traditional way to manufacture commercial high-performance rectangular waveguide lines are using machining techniques, such as Computerized Numerically Controlled (CNC) milling and Electric Discharge Machining $(\mathrm{EDM})$ in E-plane split-blocks. The strict mechanical requirements lead to the use of complex and high-precision manufacturing techniques, and thereby costly solutions for complicated structures. A corporate-fed multi-layer rectangular waveguide based slot array antenna is reported in [2]. This is based on normal rectangular waveguide technology realized by diffusion bonding of many thin copper plates in order to achieve good electrical contact between all the plates.

There exists a gap between the planar transmission lines and the non-planar hollow waveguides in terms of loss performance and fabrication flexibility. It is desired to develop a solution that has the benefits of the traditional technologies. Gap waveguide technology was proposed in [3] with promising characteristics and potential to overcome the above mentioned challenges. Several passive components, such as filters [4]-[6], planar array antennas [7]-[9], and active component packaging [10]-[12] based on gap waveguide technology have been presented over the past few years.

In this paper, we present a new type of gap waveguide technology based guiding structure, the zero-gap waveguide, to overcome the assembly problem associated with good electrical contact, especially at mm-wave frequencies. Previous works on gap waveguide [13], [14] show that at high frequencies the assembly tolerances, specially related to the gap stability, can affect the overall component performance. In the present work, a statistical analysis has been performed to study the random contact between the pin texture and the upper metal lid in order to investigate the effect of uncertain capacitances between the pins and upper plate which constitutes the gap waveguide. Also, we present a double layer slot array antenna based on this technology, which is fabricated by die-sink EDM, as a verification and application of this new technology.

\section{Zero-gap WAVEguide Structure}

The gap waveguide technology has advantageous properties, such as low loss and flexible manufacturing and assembly, 


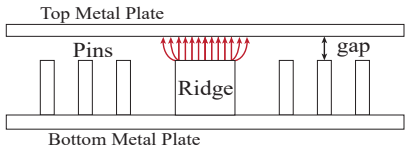

(a)

(c)

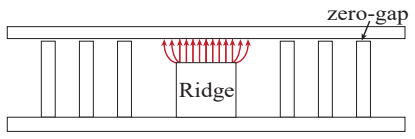

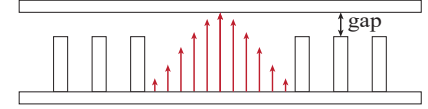

(b)

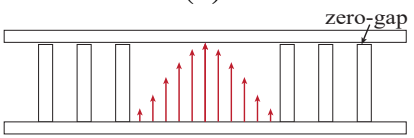

(d)
Fig. 1. Cross-sections of traditional gap waveguide structures and similar zero-gap concept. (a) Ridge gap waveguide, (b) Groove gap waveguide, (c) Ridge zero-gap waveguide, and (d) Groove zero-gap waveguide.

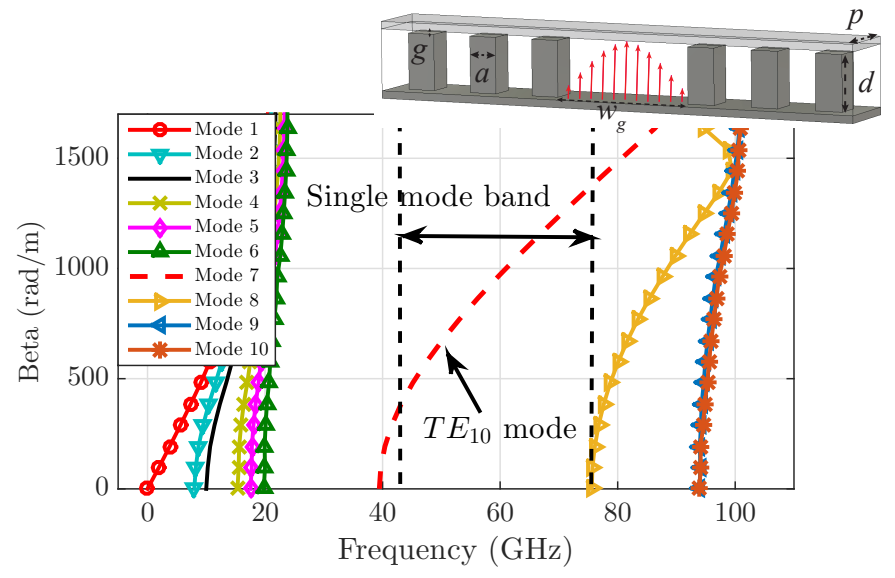

Fig. 2. Dispersion diagram for the infinite periodic unit cell including a groove in pins texture $\left(w_{g}=3.78 \mathrm{~mm}, d=1.5 \mathrm{~mm}, a=0.75 \mathrm{~mm}\right.$, $g=0.05 \mathrm{~mm}$, and $p=1.8 \mathrm{~mm}$ ).

especially at mm-wave frequencies. This new transmission line was introduced in [3] as an extension of previous works in [15] on hard and soft surfaces. The basic principle operation of gap waveguide is the use of soft/hard boundary conditions to achieve a cut-off band of all propagating modes in a PEC/PMC parallel-plate waveguide configuration. A periodic texture, e.g. a metal pins surface, in combination with a smooth metal plate creates a PEC/AMC stop-band and suppresses undesired modes and leakage [16]. It acts as a high impedance surface when the air gap between the pins and upper plate is smaller than a quarter of the wavelength. The wave propagation can be controlled by introducing a guiding structure, such as a ridge or groove in between the textured surface. However, at $\mathrm{mm}$-wave frequencies this air gap becomes quite small (about $0.25 \mathrm{~mm}$ at $60 \mathrm{GHz}$ ) and adds some constrains regarding mechanical tolerances for gap waveguide components such as antennas and filters.

Fig. 1 shows the geometry of the traditional gap waveguide structure and the equivalent zero-gap concept. In the zero-gap structure, the pins can have mechanical contact with the upper lid and thereby they can be used as a pillar for the upper metal plate assembly. The gap between the pins and upper metal plate can be made almost zero, and still maintaining the stop band properties of the pin structure. However, there is no need for electrical contact between the textured surface and upper lid. This represents a manufacturing advantage and flexible assembly technique, in particular for corporate feed-network

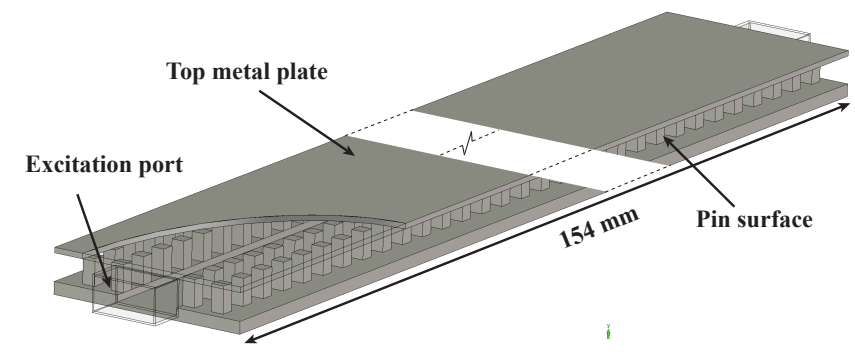

Fig. 3. Sketch of the proposed groove zero-gap waveguide line.

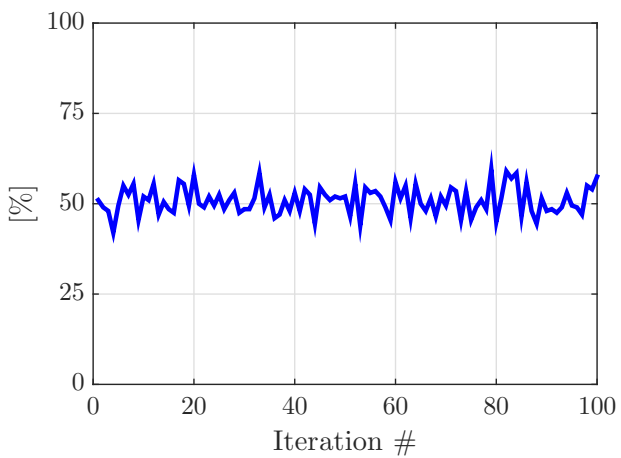

Fig. 4. Percentage of the pins that have contact with the upper plate.

of array antennas.

Therefore, the motivation of the zero-gap waveguide concept is related to the above mentioned constraint in air gap. We show in this work that the air gap between the PEC/AMC plate can be effectively reduced to almost zero and still a parallelplate stop band can be achieved similar to the traditional gap waveguide with a fixed air gap between the PEC/AMC plates.

The dispersion diagram of a unit cell, consisting of a groove structure and pins, which creates the stop-band in V-band is shown in Fig. 2. A small gap $(0.05 \mathrm{~mm})$ is considered between the pins and the upper plate. The dispersion diagram is calculated using the Eigenmode solver of CST Microwave Studio for the unit cell with periodic boundary condition. A single mode, e.g. $T E_{10}$, propagates between the two plates similar to rectangular waveguides over the frequency band 40$80 \mathrm{GHz}$.

\section{Statistical Analysis of Random Pin Contact}

Fig. 3 shows the schematic of the groove zero-gap waveguide transmission line. It consists of a textured surface and a metal plate at the top. Electrical contact between the building blocks is not needed for electrical performance, however for mechanical support, the upper metal lid can rest on the top of pin texture. Practically, in presence of flatness problem in upper metal plate, the gap between the pins and upper plate can be varied. It can happen especially in a large surface, such as feed-network of array antennas. Varying gaps can cause variable and uncertain capacitances between pins and upper plate, and thereby led to multi reflections. We study this effect to ensure the good performance of zero-gap waveguide under any circumstances. 

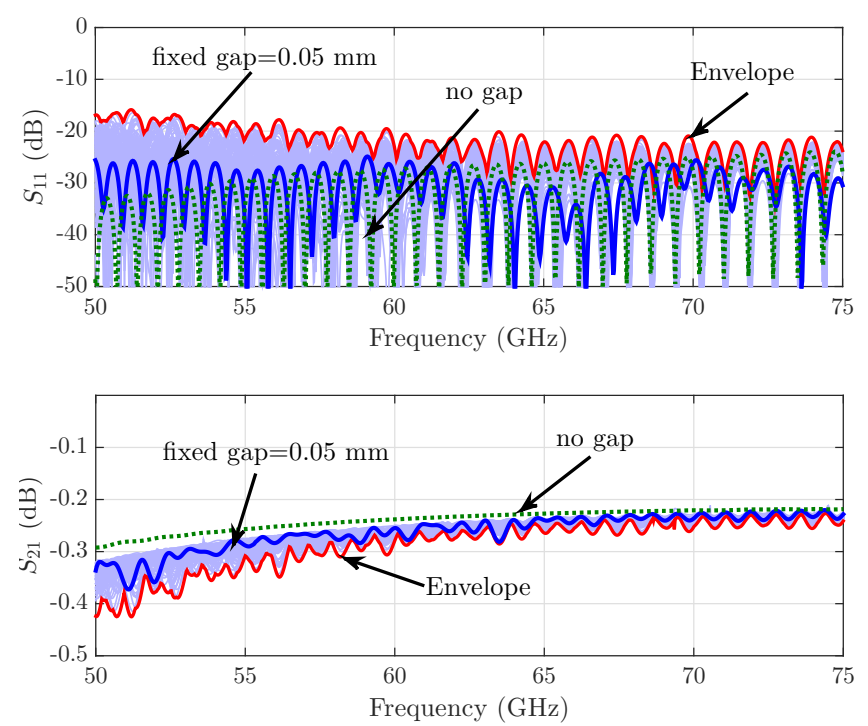

Fig. 5. Simulated S-parameters of a groove zero-gap waveguide line with 100 cases.
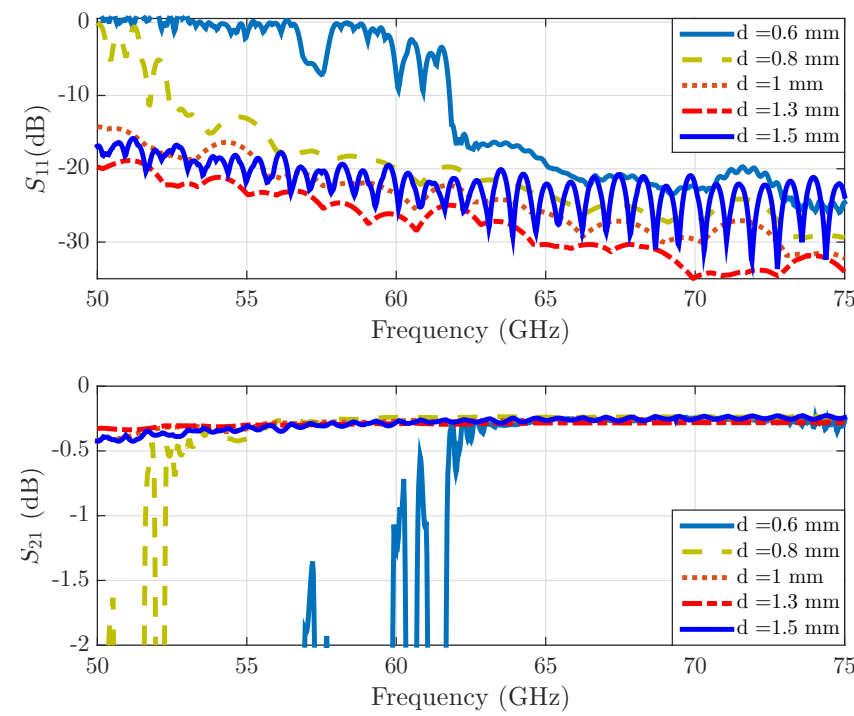

Fig. 6. The envelopes of 100 simulations of random gap for different pin heights.

We have done a statistical analysis of random contact between the pin texture and upper metal plate. The initial structure has a pin height of $1.5 \mathrm{~mm}$ with a fixed air gap of $0.05 \mathrm{~mm}$ between all pins and upper lid. A uniform random number between $0-0.1 \mathrm{~mm}$ is added to the height of each single pin, located in the two rows around the groove. Therefore, statistically $50 \%$ of the pins have electrical contact with the upper plate, as shown in Fig. 4.

Fig. 5 shows simulated results of a $154 \mathrm{~mm}$ long $(32 \lambda$ at $62.5 \mathrm{GHz})$ groove zero-gap line with 100 cases. Simulation results for the case with fixed gap equal to $0.05 \mathrm{~mm}$ for all the pins, and the case when all pins have contact with the upper plate are also presented. We have used silver as material of the structure. As shown in Fig. 5, varying the gap causes extra reflections, comparing to the case with the fixed gap.

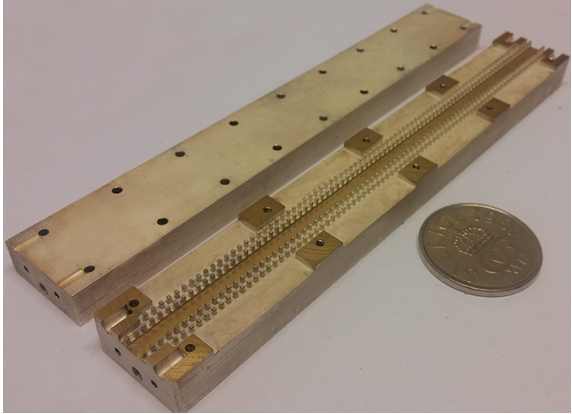

Fig. 7. Photograph of the fabricated Zero-gap waveguide straight line.
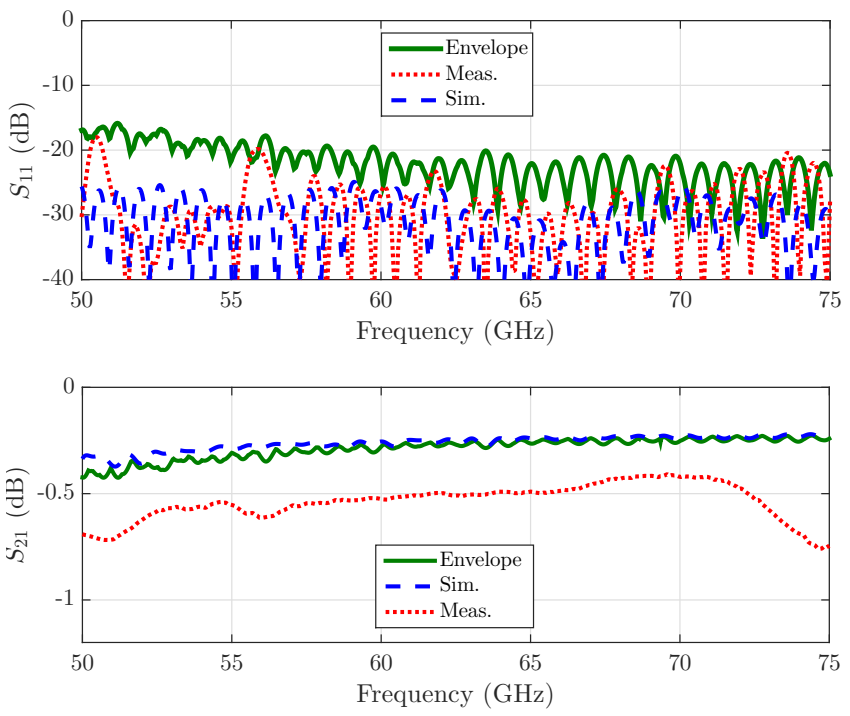

Fig. 8. Comparison of simulated and measured S-parameters of the fabricated Zero-gap waveguide prototype.

The case when all of the pins have electrical contact with the upper plate has the best performance and it is the ideal case. However, realizing the good electrical contact between the textured surface and the upper plate is very difficult and not needed in gap waveguide technology. The actual performance of the structure in Fig. 3 is expected to be below the envelope of the simulation results in Fig. 5.

Fig. 6 shows the envelopes of the S-parameters of 100 simulations with random gaps, for different pin heights. Half of the pins do not have electrical contact with the upper lid. For shorter pins, the stop-band is shifted to higher frequencies and therefore with improper stop-band the performance of the structure is degraded.

In [17] an air-filled SIW solution is presented to overcome the problem of high dielectric loss and limited power handling of substrate based transmission lines. It consists of three sandwich substrates, where an air-filled waveguide channel is made by removing the dielectric in the middle layer by using laser micromachining. A Rogers RT/Duroid 6002 substrate with thickness of $0.508 \mathrm{~mm}$ is used as middle layer substrate. It is reported that the insertion loss is reduced from 0.23 $\mathrm{dB} / \mathrm{cm}$ to $0.06 \mathrm{~dB} / \mathrm{cm}$ at U-band $(40-60 \mathrm{GHz})$. However, in this solution good electrical contact between that layers is required in order to avoid field leakage through vias. This 


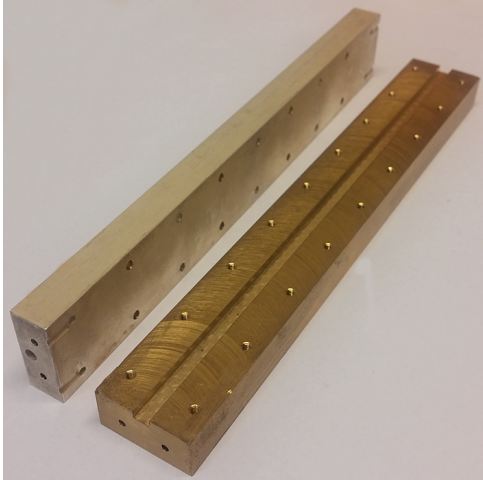

a

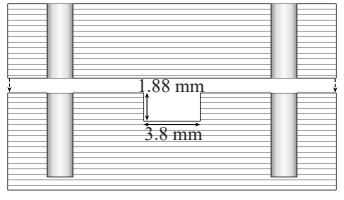

(b)

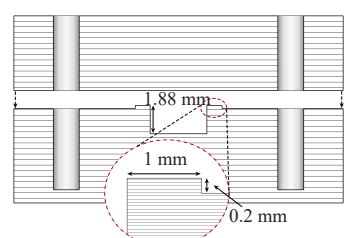

(c)
Fig. 9. (a) Photograph of the fabricated H-plane split-block rectangular waveguide. (b) Cross-section of the original line. (c) Cross-section of the modified line.

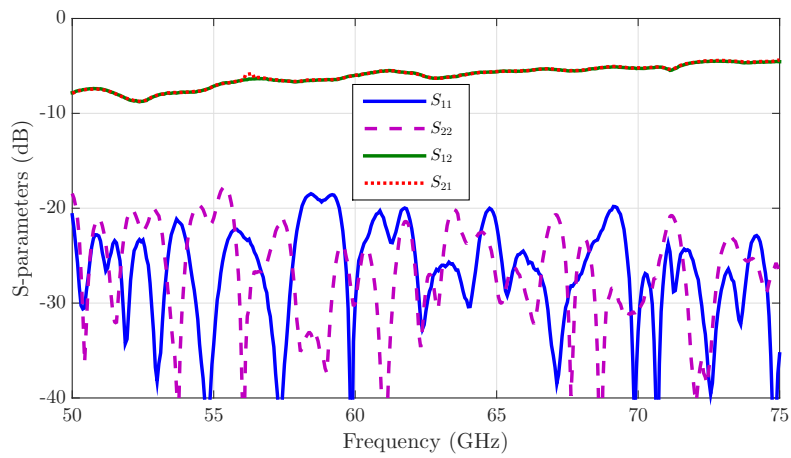

Fig. 10. Measured S-parameters of the rectangular waveguide with flat top walls.

is the main difference between gap waveguide technology and SIW. In gap waveguides the pins height is around quarter of the wavelength to produce a stop-band which eliminates any possible leakages. When the plated vias in SIW are properly implemented, the power leakage is not significant, and can be ignored. However, in SIW structures the via holes height are very short (equal to the substrate thickness and around $\lambda / 14$ at U-band in [17]) and therefore without good electrical contact there will be significant leakage.

Fig. 7 shows the fabricated zero-gap waveguide line. The prototype is fabricated in brass, and afterwards plated with silver. The measured S-parameters of the prototype is presented in Fig. 8. The measurement is carried out by using Keysight N5241A PNA-X Vector Network Analyzer and VDI WR15 extenders, with TRL (Thru, Reflect, and Line) calibration method. There is some discrepancy between the simulated result with a fixed gap and the measured $S_{11}$, which is due to uncertain gaps between the pins and top plate. However, the measured $S_{11}$ is below the envelope of statistical analysis.

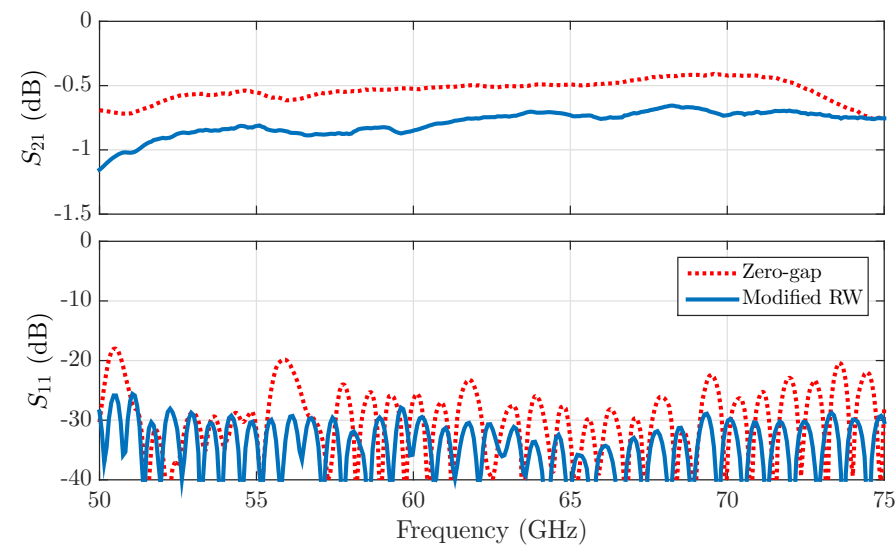

Fig. 11. Measured S-parameters of the fabricated modified rectangular waveguide.

TABLE I

COMPARISON BETWEEN DIFFERENT WAVEgUIDE TECHNOLOGIES FABRICATION AND ASSEMbly METHODS

\begin{tabular}{lcccc}
\hline \hline Ref. & Fabrication & $\begin{array}{c}\text { Freq. } \\
(\mathrm{GHz})\end{array}$ & $\begin{array}{c}\text { Split- } \\
\text { block }\end{array}$ & $\begin{array}{c}\text { Loss } \\
(\mathrm{dB} / \mathrm{cm})\end{array}$ \\
\hline$[18]$ & CNC (Al) & $75-110$ & E-plane & 0.045 \\
{$[19]$} & 3-D printing ${ }^{1}$ & $75-110$ & E-plane & 0.11 \\
Modified RW & CNC (brass) & $50-70$ & H-plane & 0.05 \\
Zero-GGW & $\begin{array}{c}\text { CNC } \\
\text { (brass+ silver plating) }\end{array}$ & $50-70$ & H-plane & 0.033 \\
\hline \hline
\end{tabular}

${ }^{1}$ Stereolithography apparatus (SLA) with copper plating.

The measured insertion loss is around $0.033 \mathrm{~dB} / \mathrm{cm}$ and it is slightly higher than the simulated one, which is due to the surface roughness and larger conductive loss of the fabricated prototype.

We have also manufactured a rectangular waveguide line with the same length in H-plane split-block. Fig. 9 (a) shows the manufactured prototype. We have used the same top metal plate for both rectangular and zero-gap waveguide prototypes. The bottom block of the fabricated rectangular waveguide is in brass. Fig. 10 shows measured S-parameters of the rectangular waveguide line with flat top walls, as shown in Fig. 9 (b). The measurement shows significant leakage due to the tiny gap between the two blocks. To improve the electrical contact between the blocks and therefore reduce the leakage, the bottom block of the prototype is modified by making small steps at the sides of the waveguide walls. The cross-section of the modified waveguide is shown in Fig. 9 (c).

The measured S-parameters of the modified waveguide is presented in Fig. 11. The measured results of the zerogap waveguide line are also presented. Adding a small step drastically improves the performance. However, the zero-gap waveguide prototype shows lower loss than the H-plane splitblock rectangular waveguide. It is worthy mentioning that the fabricated rectangular waveguide is a straight line and in present of discontinuity the performance will degrade as well.

In practice, waveguide structures are manufactured in Eplane split-blocks to avoid such leakage. In [18] an E-plane 


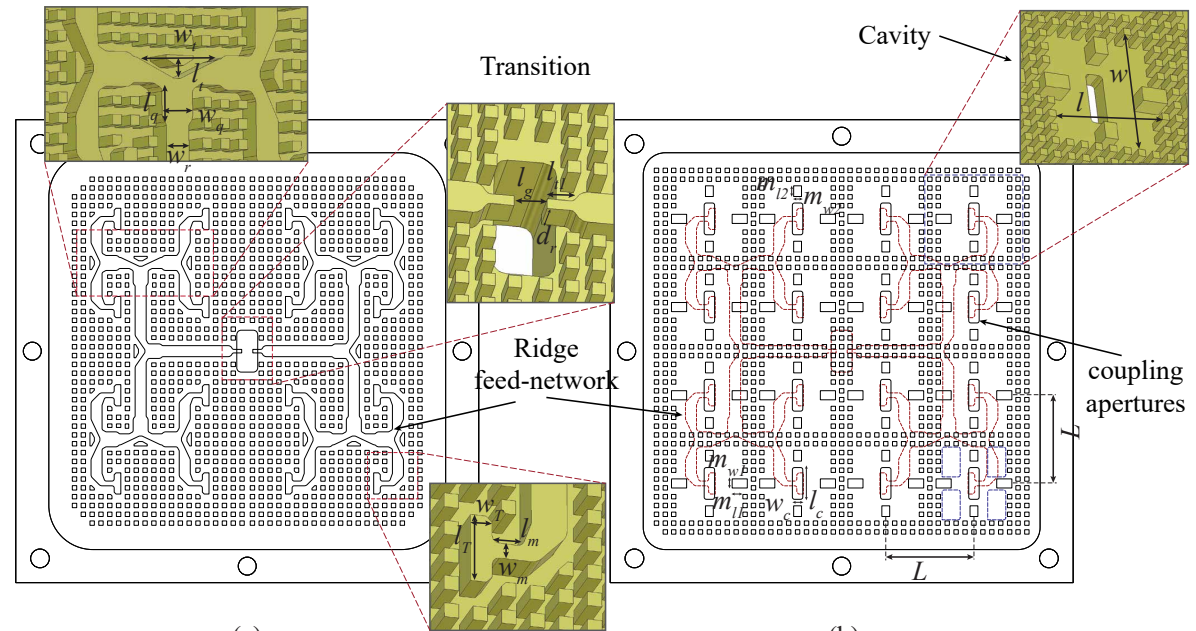

(a) (b)

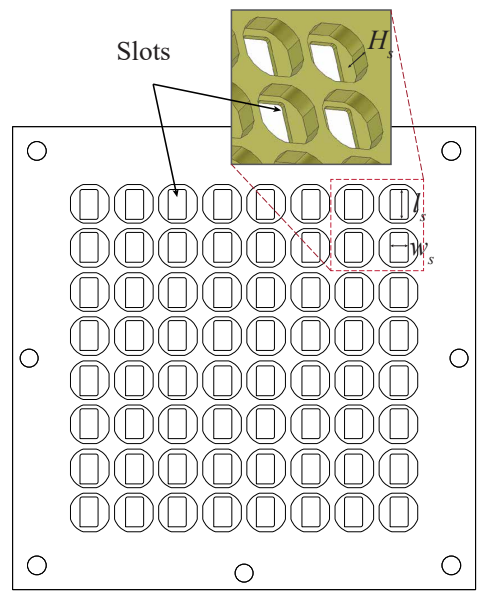

(c)

Fig. 12. Configuration of the zero-gap slot array antenna. (a) Center fed Corporate feed-network $\left(w_{r}=1 \mathrm{~mm}, w_{t}=3.5 \mathrm{~mm}, l_{t}=0.76 \mathrm{~mm}, w_{q}=1.36 \mathrm{~mm}\right.$, $l_{q}=1.2 \mathrm{~mm}, l_{t 1}=0.9 \mathrm{~mm}, l_{g}=1 \mathrm{~mm}, d_{r}=1.1 \mathrm{~mm}, w_{T}=0.6 \mathrm{~mm}, l_{T}=1.9 \mathrm{~mm}, w_{m}=0.4 \mathrm{~mm}$, and $\left.l_{m}=0.82 \mathrm{~mm}\right)$. (b) Cavity layer $(L=8 \mathrm{~mm}$, $w_{c}=0.95 \mathrm{~mm}, l_{c}=2.9 \mathrm{~mm}, m_{l 1}=1.55 \mathrm{~mm}, m_{w 1}=0.8 \mathrm{~mm}, m_{l 2}=1 \mathrm{~mm}, m_{w 2}=0.7 \mathrm{~mm}, w=6.8 \mathrm{~mm}$, and $l=6 \mathrm{~mm}$,). (c) Radiating layer with $8 \times 8$ slots $\left(w_{s}=1.6 \mathrm{~mm}, l_{s}=2.7 \mathrm{~mm}\right.$, and $\left.H_{s}=1.2 \mathrm{~mm}\right)$.

split-block rectangular waveguide is reported with average insertion loss of $0.045 \mathrm{~dB} / \mathrm{cm}$ at W-band. However, E-plane split-blocks are not really suitable for complicated wideband slot antenna arrays, such as the one presented in [2]. This mechanical flexibility of zero-gap waveguide gives an extra advantage for slot array applications and offers new opportunities for making cost-effective antennas at mm-wave frequency range.

Table I shows a comparison between different manufacturing techniques of air-filled metal rectangular waveguides. The fabricated groove zero-gap waveguide line shows a comparable performance to the E-plane split-block hollow waveguide lines, with a low insertion loss.

\section{Corporate-Fed $8 \times 8$ Slot Array Antenna}

We have designed a multilayer wideband corporate-fed slot array antenna with a ridge zero-gap waveguide distribution network to future verify the concept. The designed antenna consists of three distinct metal layers.

Fig. 12 shows the configuration of the design in more detail. A distribution feed-network is designed in the bottom layer in ridge zero-gap waveguide (Fig. 12(a)). The antenna is fed at the center by a WR-15 standard flange from the back side. A vertical transition between rectangular waveguide to ridge zero-gap waveguide is designed as an interface. $8 \times 8$ cavitybacked slots are used as radiating elements of the antenna. Each $2 \times 2$ slots on the top layer (Fig. 12(c)) are fed by a cavity in the middle layer (Fig. 12(b)). All the slots are excited with the same phase and amplitude to achieve maximum directivity. A comprehensive design procedure is presented in [20]-[22].

A prototype is manufactured by using die-sink EDM in steel with a simple mechanical assembly. The fabricated prototype is shown in Fig. 13. The total size of the antenna is $42 \mathrm{~mm}$ (length) $\times 42 \mathrm{~mm}$ (width) $\times 9 \mathrm{~mm}$ (height). In [22] the use of die-sink EDM technique to manufacture high gain

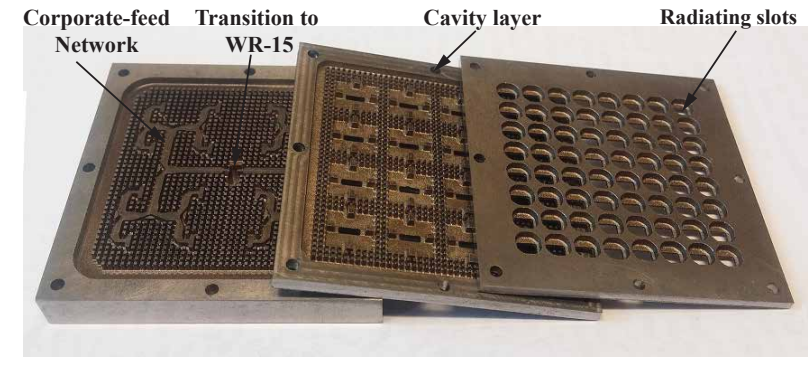

Fig. 13. Photograph of the zero-gap $8 \times 8$ slot array antenna fabricated by die-sink EDM.

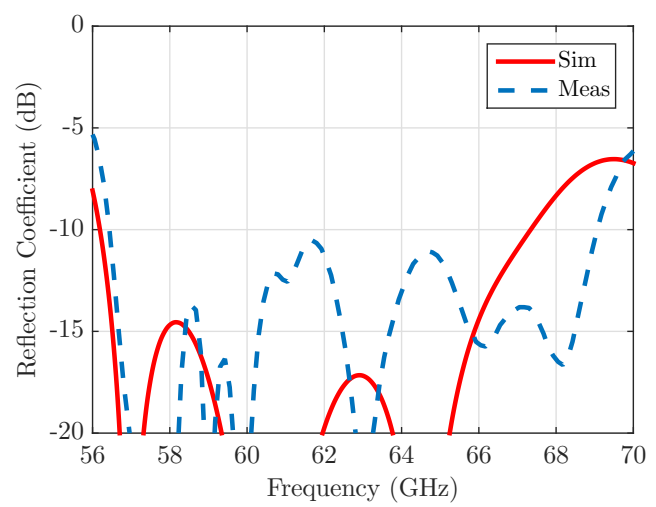

Fig. 14. Comparison of simulated and measured input reflection coefficient of the fabricated prototype.

antenna based on gap waveguide technology is presented for the first time. In EDM technique, a desired pattern is made by removing the material by high energy discharges between two conductive materials (a workpiece and an electrode). The electrode consists of the negative version of the required pattern. The electrode is usually made of graphite or copper alloys due to their high electrical and thermal conductivities, 
TABLE II

Comparison Between the Proposed and Reported Planar Array ANTENNAS

\begin{tabular}{lccccc}
\hline \hline Ref. & $\begin{array}{c}\text { Feeding } \\
\text { network }\end{array}$ & $\begin{array}{c}\text { Frequency } \\
(\mathrm{GHz})\end{array}$ & $\begin{array}{c}\text { \# of } \\
\text { elements }\end{array}$ & $\begin{array}{c}\text { Bandwidth } \\
(-10 \mathrm{~dB})\end{array}$ & $\begin{array}{c}\text { Antenna } \\
\text { efficiency }\end{array}$ \\
\hline$[23]$ & SIW & 60 & $16 \times 16$ & $15.3 \%$ & $49 \%$ \\
{$[24]$} & $\mathrm{LTCC}^{1}$ & 94 & $8 \times 8$ & $14.9 \%$ & $42 \%$ \\
{$[2]$} & $\mathrm{RW}^{2}$ & 60 & $16 \times 16$ & $12.1 \%$ & $80 \%$ \\
{$[20]$} & $\mathrm{RGW}^{3}$ & 60 & $8 \times 8$ & $14 \%$ & $65 \%$ \\
{$[22]$} & $\mathrm{RGW}^{4}$ & 60 & $16 \times 16$ & $17.6 \%$ & $60 \%$ \\
$\begin{array}{c}\text { Present } \\
\text { work }\end{array}$ & $\mathrm{RGW}^{4}$ & 60 & $8 \times 8$ & $20 \%$ & $60 \%$ \\
\hline \hline
\end{tabular}

${ }^{1}$ LTCC Ridge gape waveguide, ${ }^{2}$ Rectangular waveguide (diffusion bonding), ${ }^{3}$ Ridge gape waveguide (CNC milling) 4 Ridge gape waveguide (die-sink EDM)

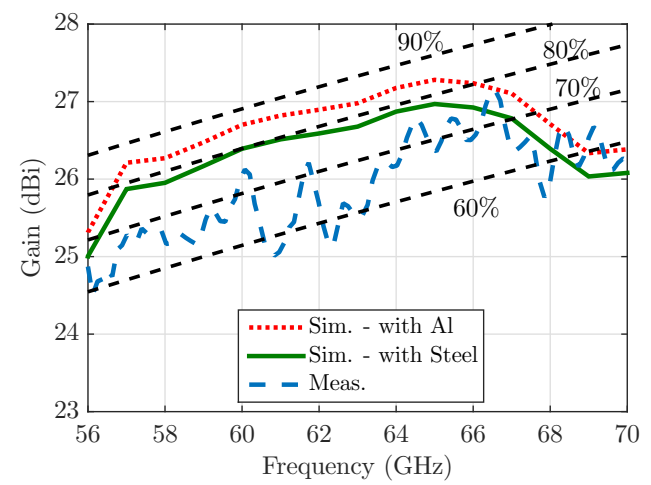

Fig. 15. Measured and computed realized gains of the designed $8 \times 8$ slot array antenna. The dashed lines are the directivities with $90 \%, 80 \%, 70 \%$, and $60 \%$ aperture efficiencies.

and high melting temperature.

Thermal and electrical conductivities of the workpiece are the most important parameters that determine the manufacturability and the quality of the final product by using EDM process. They determine how the material heats up and vaporizes. In [22] aluminum is used to fabricate the antenna with EDM technique. The thermal conductivity of aluminum is $204 \mathrm{~W} / \mathrm{mK}$. That makes aluminum a good thermal conductor and difficult to efficiently heat up locally by sparks. On the other hand, steel has lower thermal conductivity $(42.6 \mathrm{~W} / \mathrm{mK})$, which makes it a better candidate material for EDM process. We have achieved a tolerance of less than $15 \mu \mathrm{m}$ for the fabricated prototype in this paper, in contrast with the reported tolerance of $40-100 \mu \mathrm{m}$ in [22].

Fig. 14 shows the simulated and measured input reflection coefficients of the antenna. The measured reflection coefficient is below $-10 \mathrm{~dB}$ with relative impedance bandwidth of $20 \%$ ( 56.5 to $69 \mathrm{GHz}$ ). The simulated and measured realized gains of the antenna are shown in Fig. 15. The simulated realized gain for steel with electrical conductivity of $0.7 \times 10^{7} \mathrm{~S} / \mathrm{m}$ and aluminum with electrical conductivity of $3.58 \times 10^{7} \mathrm{~S} / \mathrm{m}$ are presented. The measured antenna efficiency is above $60 \%$ for most of the operating band. The reduction in gain could be due to the conductive loss and also magnetic loss of steel.

A comparison between the presented array antenna and several published planar array antennas is presented in Table II.
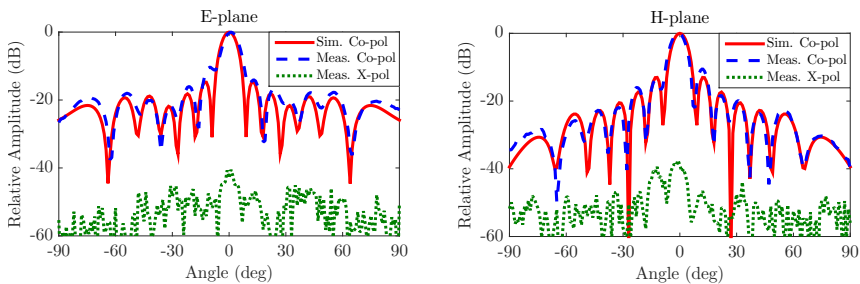

(a)
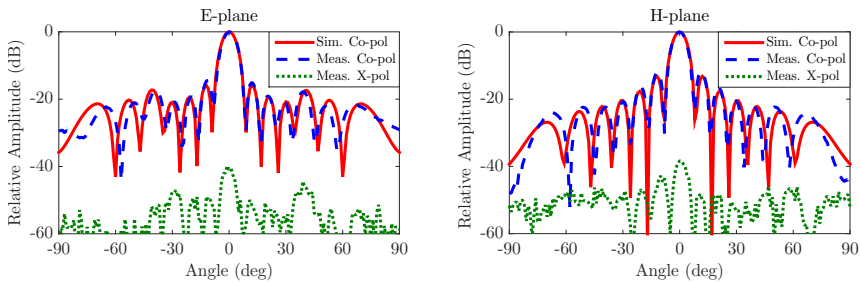

(b)
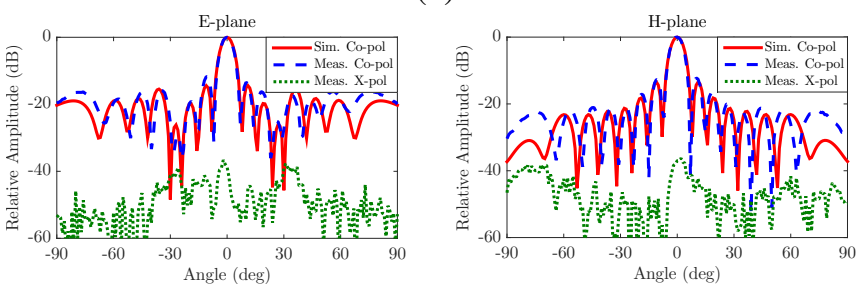

(c)

Fig. 16. Simulated and measured radiation patterns in E- and H-planes at. (a) $57 \mathrm{GHz}$, (b) $62 \mathrm{GHz}$, and (c) $68 \mathrm{GHz}$.

The SIW based corporate-fed array in [23] shows lower antenna efficiency than the hollow waveguide array antenna in [2], due to higher losses in the feed-network. A wide impedance bandwidth has been achieved with the proposed antenna. Moreover, the designed antenna shows similar efficiency to the ones in [20], [22].

The simulated and measured normalized radiation far-field patterns of the antenna in the E- and H-planes at different frequencies are presented in Fig. 16. There is a good agreement between the measured and simulated co-polar radiation pattern in a large bandwidth. The measured cross-polar patterns are also presented with cross-polarization better than $-40 \mathrm{~dB}$ in all the planes. The designed antenna exhibits good radiation pattern performance over a broad frequency range as shown in Fig. 17.

\section{CONCLUSION}

A new waveguide solution to overcome the problem of good electrical contact due to mechanical assembly constraints maintaining low loss has been presented in this paper. In this new gap waveguide structure, the pins can have mechanical contact with the upper lid and still present a good performance. Our study shows that the key characteristic of gap waveguide structures is the height of the pins, which creates a parallel-plate modes stop-band. This represents a manufacturing advantage especially for the design of complex corporate feed-network of array antennas in mm-wave frequencies. A corporate-fed $8 \times 8$ slot array antenna has been designed to show the advantage of the zero-gap concept. The measured 


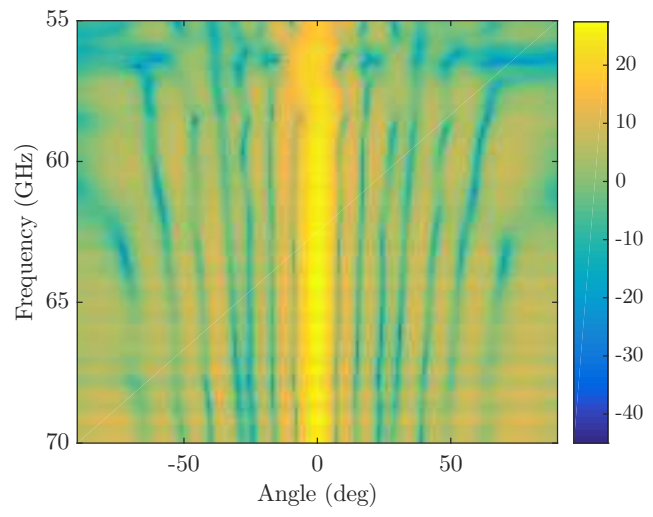

(a)

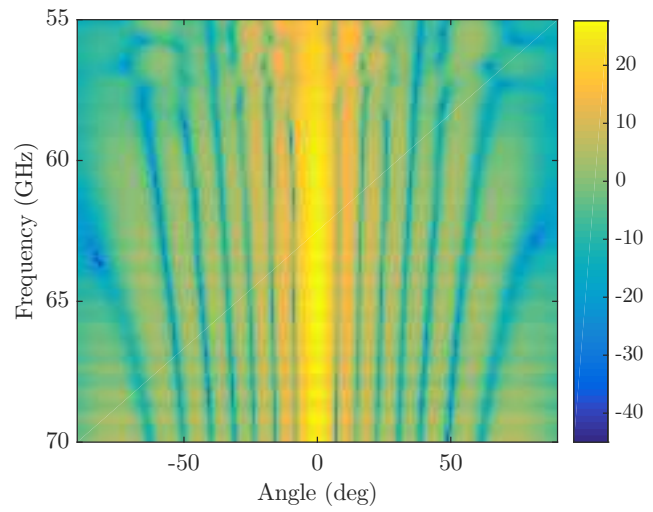

(b)

Fig. 17. Measured far-field radiation patterns versus frequency. (a) E-plane, and (b) H-plane.

results show a wide impedance bandwidth and good radiation patterns.

\section{ACKNOWLEDGMENT}

This work had been done under the supervision of the late Prof. Per-Simon Kildal. The authors would like to express their gratitude to Prof. Per-Simon Kildal for bing a source of inspiration to the members of the antenna group at Chalmers University of Technology.

\section{REFERENCES}

[1] G. Six, G. Prigent, E. Rius, G. Dambrine, and H. Happy, "Fabrication and characterization of low-loss TFMS on silicon substrate up to 220 GHz," IEEE Transactions on Microwave Theory and Techniques, vol. 53, no. 1, pp. 301-305, 2005.

[2] Y. Miura, J. Hirokawa, M. Ando, Y. Shibuya, and G. Yoshida, "Doublelayer full-corporate-feed hollow-waveguide slot array antenna in the 60GHz band," IEEE Transactions on Antennas and Propagation, vol. 59, no. 8, pp. 2844-2851, Aug. 2011.

[3] P.-S. Kildal, E. Alfonso, A. Valero-Nogueira, and E. Rajo-Iglesias, "Local metamaterial-based waveguides in gaps between parallel metal plates," IEEE Antennas and Wireless Propagation Letters, vol. 8, pp. 84-87, 2009.

[4] A. Vosoogh, A. A. Brazález, and P.-S. Kildal, "A V-band inverted microstrip gap waveguide end-coupled bandpass filter," IEEE Microwave and Wireless Components Letters, vol. 26, no. 4, pp. 261-263, Apr. 2016.

[5] E. A. Alós, A. U. Zaman, and P.-S. Kildal, "Ka-band gap waveguide coupled-resonator filter for radio link diplexer application," IEEE Transactions on Components, Packaging and Manufacturing Technology, vol. 3, no. 5, pp. 870-879, 2013.
[6] M. S. Sorkherizi and A. A. Kishk, "Fully printed gap waveguide with facilitated design properties," IEEE Microwave and Wireless Components Letters, vol. 26, no. 9, pp. 657-659, 2016.

[7] S. A. Razavi, P.-S. Kildal, L. Xiang, E. Alfonso Alos, and H. Chen, " $2 \times 2$-slot element for $60-\mathrm{GHz}$ planar array antenna realized on two doubled-sided pcbs using SIW cavity and EBG-type soft surface fed by microstrip-ridge gap waveguide," IEEE Transactions on Antennas and Propagation, vol. 62, no. 9, pp. 4564-4573, Sep. 2014.

[8] H. Attia, M. S. Sorkherizi, and A. A. Kishk, " $60 \mathrm{GHz}$ slot antenna array based on ridge gap waveguide technology enhanced with dielectric superstrate," in Proceedings of the 9th European Conference on Antennas and Propagation (EuCAP). Lisbon, 2015, pp. 1-4.

[9] M. S. Sorkherizi, A. Dadgarpour, and A. A. Kishk, "Planar highefficiency antenna array using new printed ridge gap waveguide technology," IEEE Transactions on Antennas and Propagation, vol. 65, no. 7, pp. 3772-3776, 2017.

[10] A. A. Brazález, A. U. Zaman, and P.-S. Kildal, "Improved microstrip filters using PMC packaging by lid of nails," IEEE Transactions on Components, Packaging and Manufacturing Technology, vol. 2, no. 7, pp. 1075-1084, 2012.

[11] B. Ahmadi and A. Banai, "Substrateless amplifier module realized by ridge gap waveguide technology for millimeter-wave applications," IEEE Transactions on Microwave Theory and Techniques, vol. 64, no. 11, pp. 3623-3630, 2016.

[12] U. Nandi, A. U. Zaman, A. Vosoogh, and J. Yang, "Novel millimeter wave transition from microstrip line to groove gap waveguide for MMIC packaging and antenna integration," IEEE Microwave and Wireless Components Letters, vol. 27, no. 8, pp. 691-693, 2017.

[13] A. A. Brazález, E. Rajo-Iglesias, J. L. Vazquez-Roy, A. Vosoogh, and P.S. Kildal, "Design and validation of microstrip gap waveguides and their transitions to rectangular waveguide, for millimeter-wave applications," IEEE Transactions on Microwave Theory and Techniques, vol. 63, no. 12, pp. 4035-4050, 2015.

[14] A. A. Brazález, J. Flygare, J. Yang, V. Vassilev, M. Baquero-Escudero, and P.-S. Kildal, "Design of F-band transition from microstrip to ridge gap waveguide including Monte Carlo assembly tolerance analysis," IEEE Transactions on Microwave Theory and Techniques, vol. 64, no. 4 pp. 1245-1254, 2016.

[15] P.-S. Kildal, "Artificially soft and hard surfaces in electromagnetics," IEEE Transactions on Antennas and Propagation, vol. 38, no. 10, pp. $1537-1544,1990$.

[16] E. Rajo-Iglesias and P.-S. Kildal, "Numerical studies of bandwidth of parallel-plate cut-off realised by a bed of nails, corrugations and mushroom-type electromagnetic bandgap for use in gap waveguides," IET Microwaves, Antennas and Propagation, vol. 5, no. 3, pp. 282289, 2011.

[17] F. Parment, A. Ghiotto, T.-P. Vuong, J.-M. Duchamp, and K. Wu, "Air-filled substrate integrated waveguide for low-loss and high powerhandling millimeter-wave substrate integrated circuits," IEEE Transactions on Microwave Theory and Techniques, vol. 63, no. 4, pp. 12281238, 2015.

[18] I. Stil, A. Fontana, B. Lefranc, A. Navarrini, P. Serres, and K. Schuster, "Loss of WR10 waveguide across 70-116 GHz," in Proceedings of the 22nd International Symposium on Space Terahertz Technology, 2012, pp. 1-3.

[19] M. D'Auria, W. J. Otter, J. Hazell, B. T. Gillatt, C. Long-Collins, N. M. Ridler, and S. Lucyszyn, "3-D printed metal-pipe rectangular waveguides," IEEE Transactions on Components, Packaging and Manufacturing Technology, vol. 5, no. 9, pp. 1339-1349, 2015.

[20] A. Vosoogh and P.-S. Kildal, "Corporate-fed planar $60 \mathrm{GHz}$ slot array made of three unconnected metal layers using AMC pin surface for the gap waveguide," IEEE Antennas and Wireless Propagation Letters, vol. 15, pp. 1935-1938, Dec. 2015.

[21] D. Zarifi, A. Farahbakhsh, A. U. Zaman, and P.-S. Kildal, "Design and fabrication of a high-gain $60-\mathrm{GHz}$ corrugated slot antenna array with ridge gap waveguide distribution layer," IEEE Transactions on Antennas and Propagation, vol. 64, no. 7, pp. 2905-2913, 2016.

[22] A. Vosoogh, A. U. Zaman, and V. Vassilev, "Wideband cavity-backed slot subarray with gap waveguide feed-network for D-band applications," in Proceedings of the 11th European Conference on Antennas and Propagation (EuCAP). Paris, 2017, pp. 207-209.

[23] Y. Li and K.-M. Luk, "60-GHz substrate integrated waveguide fed cavity-backed aperture-coupled microstrip patch antenna arrays," IEEE Transactions on Antennas and Propagation, vol. 63, no. 3, pp. 10751085, Mar. 2015.

[24] B. Cao, H. Wang, Y. Huang, and J. Zheng, "High-gain L-probe excited substrate integrated cavity antenna array with LTCC-based gap waveg- 
uide feeding network for W-band application," IEEE Transactions on Antennas and Propagation, vol. 63, no. 12, pp. 5465-5474, 2015.

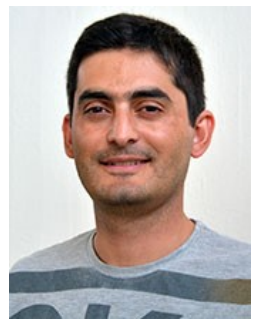

Abbas Vosoogh received the B.Sc. degree in electrical engineering from the University of Sistan and Baluchestan, Zahedan, Iran, and the M.Sc. degree from K. N. Toosi University of Technology, Tehran, Iran, in 2008 and 2011, respectively. He is currently pursuing his Ph.D. degree with Chalmers University of Technology, Gothenburg, Sweden. His current research interests include the development of gap waveguide technology for millimeter and submillimeter wave applications, microwave passive components, EBG, soft and hard surfaces, mm-wave planar array antennas, and integration of passive and active components with array antenna.

Mr. Vosoogh was a recipient of the Best Student Paper Award of the 2015 International Symposium on Antennas and Propagation, TAS, Australia, the CST University Publication Award 2016, the Best Paper Award and the Best Student Paper Award of the 2016 International Symposium on Antennas and Propagation, Okinawa, Japan, and the First Prize Student Award of the 2017 IEEE International Symposium on Antennas and Propagation and USNCURSI Radio Science Meeting, San Diego, CA, USA.

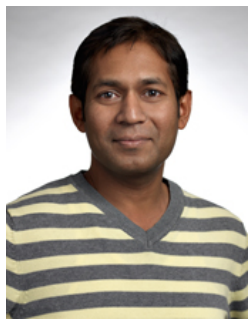

Ashraf Uz Zaman (M'14) was born in Chittagong, Bangladesh. He received the B.Sc. degree in electrical and electronics engineering from the Chittagong University of Engineering and Technology, Chittagong. He received the M.Sc. and $\mathrm{PhD}$ degree from Chalmers University of Technology, Gteborg, Sweden, in 2007 and 2013 respectively. He is currently an assistant professor with the Communication and Antenna Division of the same university. His current research interests include millimeter wave high efficiency planar antennas in general, gap waveguide technology, frequency selective surfaces, microwave passive components, packaging techniques and integration of MMICs with the antennas.

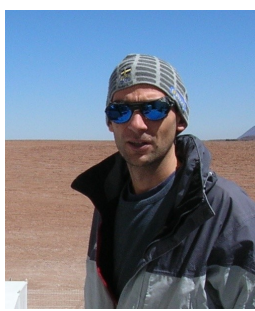

Vessen Vassilev received M. Sc degrees in Radio Communications from the Sofia Technical University in 95, and in Digital Communications from Chalmers University of Technology in 98. In 2003 he received his $\mathrm{PhD}$ degree from the department of $\mathrm{Ra}-$ dio and Space Science at Chalmers University. Between 1998 and 2008 he has been working with the development of mm-wave receivers for applications in radio astronomy and space sciences. Instruments designed by him are currently in operation at the Atacama Pathfinder Experiment (APEX) telescope and at the Onsala Space Observatory. Since 2008 he has been with the Microwave Electronics Laboratory at the Department of Microtechnology and Nanoscience at Chalmers. His current interests are in the development mmwavelength sensors based on MMIC technologies.

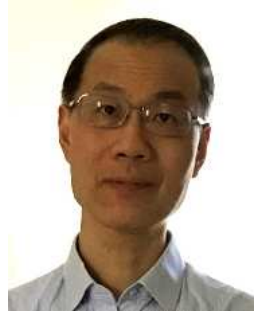

Jian Yang (M'02SM'10) received the B.Sc. degree in electrical engineering from the Nanjing University of Science and Technology, Nanjing, China, in 1982, the M.Sc. degree in electrical engineering from the Nanjing Research Center of Electronic Engineering, Nanjing, in 1985, and the Swedish Licentiate and $\mathrm{Ph} . \mathrm{D}$. degrees from Chalmers University of Technology, Gothenburg, Sweden, in 1998 and 2001, respectively. From 1985 to 1996 , he was with the Nanjing Research Institute of Electronics Technology, Nanjing, China, as a Senior Engineer. From 1999 to 2005, he was with the Department of Electromagnetics, Chalmers University of Technology, Gothenburg, Sweden, as a Research Engineer. During 2005 and 2006, he was with COMHAT AB as a Senior Engineer. From 2006 to 2010, he was an Assistant Professor, from 2010 to 2016, he was an Associate Professor, and since 2016 he has been a professor with the Department of Signals and Systems, Chalmers University of Technology. His research interests include $60-140 \mathrm{GHz}$ antennas, terahertz antennas, MIMO antennas, ultrawideband (UWB) antennas and UWB feeds for reflector antennas, UWB radar systems, UWB antennas in near-field sensing applications, hat-fed antennas, reflector antennas, radome design, and computational electromagnetics. 